\title{
Physical Properties and Evolutionary Stage of Be Stars
}

\author{
P. Harmanec ${ }^{1}$ \\ Astronomical Institute, Academy of Sciences of the Czech Republic, \\ CZ-251 65 Ondřejov, Bohemia - Czech Republic
}

\begin{abstract}
The determination of accurate and reliable basic physical properties of Be stars is a very complicated task which, among other things, requires a good knowledge of the variability pattern of each particular object. Possible confusion between a rotationally distorted photosphere and a pseudophotosphere - an inner optically thick part of a gradually formed envelope which temporarily mimics a photosphere - is discussed in some detail in relation to the stellar effective temperature, brightness and rate of rotation. It is shown that the currently available data allow us to set relatively stringent limits on possible radii of at least some brighter Be stars, but very few direct determinations of mass have been made. The only exceptions are the Be stars in eclipsing binaries. The trouble is, however, that all of them are interacting binaries for which reliable orbital solutions are seriously hampered by the presence of gas streams and by strong interactions between the binary components. Moreover, their masses need not be representative for the whole population of Be stars.

An answer to the question about the evolutionary stage of Be stars is even more uncertain since it is closely related to the principal, as yet unanswered question about the origin of the Be phenomenon itself.
\end{abstract}

\section{Introduction and the concept of a pseudophotosphere}

The crucial property of Be stars is their variability on a number of time scales; especially pronounced are the long-term $\mathrm{B} \rightarrow \mathrm{Be} \rightarrow \mathrm{Be}$ shell transitions which usually occur on a timescale from years to decades.

Two types of correlation between spectral changes and light variations, positive and inverse correlation, were first explicitly recognized by Dachs (1982) and later described in detail and interpreted by Harmanec $(1983,1994)$. For the positive correlation, a brightening of the object occurs at the beginning of a new emission-line phase. At the same time, the Be star moves from the main sequence towards the supergiant sequence in the $U-B$ vs. $B-V$ diagram, more or less preserving its photometric spectral class. For the inverse correlation, a light decrease is observed at the beginning of a new emission episode and the

${ }^{1}$ Current Address: Institute of Astronomy, Faculty of Mathematics and Physics of the Charles University, V Holešovičkách 2, CZ-180 00 Praha 8, Bohemia - Czech Republic 
Be star moves along the main sequence towards a later spectral subclass in the $U-B$ vs. $B-V$ diagram.

Harmanec (1983) introduced the concept of a pseudophotosphere by which he means the inner parts of the Be envelope which are flattened towards the stellar equator and optically thick in continuum. He then explains the two types of correlation by an aspect effect: If the star is seen roughly equator-on, the (cooler than star) pseudophotosphere is shielding partly the stellar photosphere and the object appears fainter and cooler. If the Be star is seen under some arbitrary inclination, significantly different from the equator-on view, the formation of the pseudophotosphere (which redistributes the stellar radiation away from the equatorial plane) acts as an increase of the effective radius of the star. One therefore observes a brightening, and also a change of the apparent luminosity class of the object from a dwarf towards a (super)giant. (Compare that to a somewhat different interpretation, put forward by Hirata 1995, which is based on Collins' et al. 1991 model photospheres of rotating stars.) There are several findings supporting Harmanec's interpretation: (1) The positive correlation referred to above is observed more frequently than the inverse one, as expected. (2) Hubert and Floquet (1998) concluded from the light curves of a number of Be stars observed by Hipparcos that the inverse correlation occurs for stars with high $v \sin i$. (3) Geometrical interpretation is also supported by the fact that any given Be star shows always the same type of long-term behaviour - either brightenings or fadings - cf., e.g., Harmanec (1998a). (4) Interpretation in terms of a pseudophotosphere has also been supported by the recent comparison of observed and model spectra of V839 Her - see Koubský et al. (1997).

\subsection{Principal Problems in Deriving Stellar Properties of Be Stars}

A. The presence of Be envelopes and rapid rotation affect both, the line and the continuum spectrum of the star. Besides, one has to distinguish between the pseudophotosphere and rotationally distorted atmosphere of the star; geometry and darkening laws of the pseudophotosphere can be quite different from that of the photosphere. Hadrava (1992) showed that even for static grey atmospheres the theorem by von Zeipel need not hold. This in turn means that the gravity darkening may in fact be smaller than that usually assumed. All these complications imply that it is not trivial to derive meaningful spectral and luminosity class and appropriate stellar $T_{\text {eff }}$ for any particular Be star.

B. Time variability of line and continuum spectrum also makes the determination of the visual magnitude of a Be star difficult: One cannot simply use the $V$ magnitude of the object obtained at some arbitrary epoch: Instead, one needs to know the observational history of the star and the type of the correlation between the brightness and emission strength, discussed above. Clearly, the best choice is to adopt the visual magnitude from the period when the star is apparently without emission but even this is not a completely secure procedure. Note that the "undisturbed" level of X Per was not always the same between the consecutive emission-line episodes (cf., e.g., Piccioni et al. 2000, Roche et al. 2000). Note also that the statistical studies of Be stars in clusters can be systematically affected: For faint objects, the observers mainly detect stars with a strong Balmer emission. The observed magnitudes and colours of such objects are inevitably peculiar and do not characterize well the underlying stars. Since 
the positive correlation is statistically more frequent than the inverse one, the majority of the investigated Be stars will appear brighter than they are. This effect may be partly responsible for the often repeated statement that the $\mathrm{Be}$ stars are on average brighter than their non-emission counterparts.

C. For most of the Be stars, there is almost no direct information on the inclination of their rotational axes and on the latitudinal dependence of their surface temperatures caused by rapid rotation. This constitutes an additional uncertainty concerning their spectral class. Also the apparent luminosity class can be affected, both in broad-band colours and in the line profiles - see, e.g., the He I line profiles of 60 Cyg from different epochs (Koubský et al. 2000).

\section{Radii and masses of Be stars}

This section presents data on masses and radii of some particular Be stars which could be derived with some confidence from the existing observations. Since the origin of the Be phenomenon remains unclear, I prefer to adopt the original phenomenological definition of a Be star, based on the observed presence of the Balmer emission. I attempted to derive radii and masses for a selection of bright emission-line stars of all apparent luminosity classes for which good observational data are available.

\subsection{Methods used}

There are only a few methods for obtaining radii and masses of Be stars.

A direct determination of mass and radius This is certainly the most accurate method. However, it can only be used for Be stars which are members of eclipsing binary systems. The trouble is that virtually all known eclipsing binaries containing Be stars are interacting binaries. This complicates reliable determination of their basic properties. Besides, one can ask if their masses and radii are representative for the general population of Be stars. Arne Slettebak writes in his Boulder review (Stettebak 1987): "Be stars are not found as components in well-detached systems, while semi-detached systems yield values which are difficult to interpret." This is still true, although the progress of methods of analyzing light and RV curves of systems with circumstellar accretion disks led to several determinations of Be-star masses and radii which can be adopted with some confidence.

Radii derived from the definition of the effective temperature and the distance modulus: Although known for a long time, this method can now be applied with confidence to a number of Be stars thanks to the accurate parallaxes, derived by Hipparcos (Perryman et al. 1997). One has to use properly dereddened observed visual magnitude $V_{0}$, estimated $T_{\text {eff }}$, bolometric correction $\mathrm{BC}$ (from Code et al. 1976) and parallax $\pi$ (in seconds of arc). The working equation is:

$$
\log R / R_{\odot}=7.474-2 \log T_{\text {eff }}-0.2 \cdot B C-0.2 \cdot V_{0}-\log \pi .
$$

As mentioned above, some uncertainty is associated with the proper value of $T_{\text {eff }}$ for Be stars. Fortunately, the function $2 \log T_{\text {eff }}+0.2 B C$ varies only slowly 

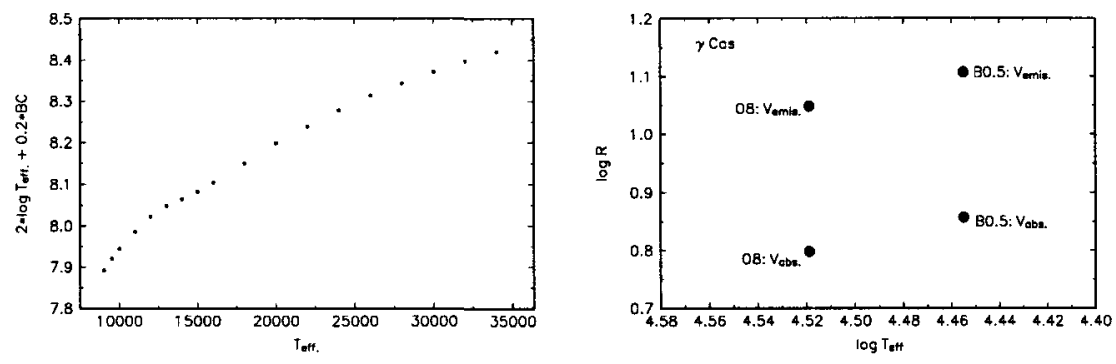

Figure 1. Left: Function $2 \log T_{\text {eff }}+0.2 B C$ plotted vs. $T_{\text {eff }}$ over the range corresponding to spectral types from $\mathrm{O} 8$ to A2. Right: Radius of $\gamma$ Cas derived for two choices of $T_{\text {eff }}$ and for the extreme values of the observed $V$ magnitude of the star

along the B sequence as illustrated by the left panel of Fig. 1 which means that even an approximate estimate of $T_{\text {eff }}$ should lead to a rather accurate value of the radius if a good value of parallax is available. To check on how this method compares with a direct determination, I used the Hipparcos parallax, $T_{\text {eff }}$ and observed $V$ of $\mathrm{U}$ Cep to obtain $R=2.59(2.33-2.91) \mathrm{R}_{\odot}$, in a complete agreement with the direct determination of $R=2.69 \mathrm{R}_{\odot}$. As another check, I calculated the radius of $\gamma$ Cas for two possible spectral types (see below) and for the maximum and minimum observed $V$ magnitude of the star. The results are shown in the right panel of Fig. 1. Obviously, the radius is rather insensitive to the exact choice of the effective temperature. However, the use of the $V$ magnitude from a strong emission-line phase can lead to about a factor of two error in the radius.

Radii derived from the parallax and interferometrically measured angular diameter In a few cases of bright emission-line stars, their angular diameters $\vartheta$ (in seconds of arc) are available from the intensity interferometry - see Code et al. (1976). Their linear radii then follow from: $R / R_{\odot}=107.47 \cdot \vartheta \pi^{-1}$.

The uncertainty in Be-star masses There is currently no suitable method of indirect determination of masses of Be stars and one has to assume normal masses corresponding to their effective temperatures, for instance adopting the relations from Harmanec's (1988) study. Perhaps the most promising way of obtaining the first reliable data on the Be-star masses and also an independent estimate of the parallax is to observe RV curves of Be stars and their companions in visual binaries. Suitable candidates are systems with short enough orbital periods which also have good visual orbital elements - inclination in particular - derived from speckle interferometry. An excellent candidate is $\delta$ Sco which will be passing though the periastron of its 10.5-yr orbit during the year 2000 . According to Bedding (1993), the secondary is $1 \mathrm{~m}_{5}$ fainter than the primary in $V$ which means that its lines could be observed and the RV curve derived if a good disentangling like KOREL (Hadrava 1995) would be applied. 


\subsection{Real estimates of Be-star masses and radii}

Masses and radii of Be stars in eclipsing binaries I used only determinations in which the effects of the circumstellar matter were taken into account. For $U \mathrm{Cep}$, I combined photometric and spectroscopic elements from various sources myself. The basic physical properties of emission-line components are summarized in Table 1. (Any possible omissions are caused solely by my ignorance.)

Table 1. The basic physical properties of emission-line stars which are members of eclipsing binaries

\begin{tabular}{rrcccrl}
\hline Star & HD & $\log T_{\text {eff }}$ & $\begin{array}{c}R \\
\left(\mathrm{R}_{\odot}\right)\end{array}$ & $\begin{array}{c}M \\
\left(\mathrm{M}_{\odot}\right)\end{array}$ & $\begin{array}{r}M_{\text {bol }} \\
(\mathrm{mag} .)\end{array}$ & Sources \\
\hline U Cep & 5679 & 4.112 & 2.69 & 4.21 & -0.90 & $1,2,3$ \\
RY Per & 17034 & 4.261 & 4.06 & 6.25 & -3.28 & 4 \\
RY Gem & 58713 & 3.973 & 2.5 & 2.36 & 0.65 & 5 \\
AI Cru & - & 4.384 & 4.95 & 10.3 & -4.94 & 6 \\
TX UMa & 99033 & 4.110 & 2.83 & 4.76 & -1.00 & 7 \\
V360 Lac & 216200 & 4.280 & 8.8 & 6.5 & -5.15 & 8 \\
\hline
\end{tabular}

Sources: 1. Batten (1974), 2. Tomkin (1981), 3. Olson (1984), 4. Olson \& Plavec (1997), 5. Plavec \& Dobias (1987), 6. Bell et al. (1987), 7. Maxted et al. (1995), 8. Hill et al. (1997)

Radii based on measured parallaxes Currently the most accurate parallaxes for Be stars come from the properly derived mean parallaxes of not too distant open clusters containing Be stars. Pinsonneault et al. (1998) pointed out a rather large discrepancy between the Hipparcos parallax of Pleiades and that derived from photometry and main-sequence fitting. They warned that there may be systematic errors in the Hipparcos parallaxes. However, the consecutive studies by van Leeuwen (1999) and Robichon et al. (1999) showed that such errors seem unlikely. I therefore based my estimates on the Hipparcos parallaxes. For Be stars in open clusters and associations I used the mean cluster parallaxes and $E(B-V)$ to obtain probably the most accurate radii derived by this method.

The practical application was by no means easy and there is not enough space to describe it in detail. For each star, I investigated its long-term photometric behaviour, using mainly the Hvar photometry (Pavlovski et al. 1997), the Hipparcos $H_{p}$ magnitudes transformed to Johnson $V$ according to Harmanec (1998b) and $U B V$ photometry secured by Percy and his collaborators (Percy, priv. com.). Consulting the shape of the light curves and spectroscopic records in the literature, I identified the phases when the star was without emission and adopted the corresponding $V$ magnitude as characteristic for the star itself. Also the reddening was derived for the phases without emission. I usually consulted the $U-B$ vs. $B-V$ diagram and several other independent determinations (e.g. Beeckmans \& Hubert-Delplace 1980) whenever possible. The effective temperature was estimated for non-emission phases using the procedures by Moon and Dworetsky (1985) if no direct analysis of the spectra was available. For the 
Table 2. Radii of emission-line stars for which angular diameters from intensity interferometry are given by Code et al. (1976): Hippar$\cos$ parallaxes are used. $R_{1}$ denotes the radius derived using $\log T_{\text {eff }}$ and $V_{0}$ while $R_{2}$ is the radius based on the measured angular diameter. The ranges of possible radii, estimated from the errors of the parallaxes, are given in brackets

\begin{tabular}{rcrccc}
\hline HD & $\log T_{\text {eff }}$ & $V_{0}$ & $M_{\text {bol }}$ & $R_{1}$ & $R_{2}$ \\
\hline 10144 & 4.162 & .450 & -4.024 & $9.01(8.79-9.24)$ & $9.10(8.55-9.67)$ \\
34085 & 4.063 & .205 & -7.264 & $63.22(53.04-78.24)$ & $64.94(53.41-81.94)$ \\
37128 & 4.395 & 1.480 & -9.075 & $31.55(22.95-50.43)$ & $30.52(20.91-51.61)$ \\
37742 & 4.476 & 1.950 & -7.950 & $12.94(10.80-16.14)$ & $12.93(9.89-17.46)$ \\
66811 & 4.623 & 2.107 & -9.797 & $15.40(12.63-19.71)$ & $19.37(14.76-26.57)$ \\
68273 & 4.544 & 1.970 & -8.371 & $11.49(10.11-13.30)$ & $12.19(9.50-15.72)$ \\
143275 & 4.498 & 2.067 & -6.761 & $6.76(6.67-6.86)$ & $7.16(6.45-7.90)$ \\
149757 & 4.504 & 1.607 & -7.185 & $8.00(7.27-8.89)$ & $7.70(6.31-9.39)$ \\
\hline
\end{tabular}

emission-line stars studied by Code et al. (1976), I also consulted their $T_{\text {eff }}$ and $E(B-V)$ values. The results of my determinations are summarized in two tables. Table 2 compares the results of two partly independent determinations for stars also studied by Code et al. (1976). The agreement is good, always within the quoted errors. The radii for Be stars which are members of open clusters or associations with good mean parallaxes are in the first part of Table 3 . These should also be quite reliable. Finally, the radii of some field emission-line stars are given in the second part of Table 3 . They are based on their individually measured Hipparcos parallaxes.

\subsection{Discussion of a few often studied Be stars}

$\gamma$ Cas (HR 264, HD 5394) is a crucial but complicated object. Its recorded brightness in V ranges from $1^{\mathrm{m}} 65$ to $2^{\mathrm{m}} 9$ (Howarth 1979). Hutchings (1970) modelled its rotationally broadened Balmer profiles and obtained $T_{\text {pole }}=(33000$ $\pm 3000) \mathrm{K}, T_{\text {equ. }}=(12000 \pm 200) \mathrm{K}$, inclination $i:(55 \pm 10)$ degrees, $R_{e}=$ $6.5 \mathrm{R}_{\odot}$, i.e. an $\mathrm{O} 8$ star rotating at break-up. Poeckert and Marlborough (1978) adopted $M=17 \mathrm{M}_{\odot}, R=10 \mathrm{R}_{\odot}, T_{\text {eff }}=25000 \mathrm{~K}, i=45^{\circ}$ in their well-known model. Quirrenbach et al. (1997) derived the axial ratio of the tilted envelope interferometrically to be $(0.70 \pm 0.02)$ and the disk radius $(3.47 \pm 0.02)$ mas. This implies $i>44^{\circ}$. My analysis gives a radius between 5.7 and $7.0 \mathrm{R}_{\odot}$ while using the $V$ magnitude from the emission phase would lead to $10.1-12.5 \mathrm{R}_{\odot}$. The true $v \sin i$ is certainly closer to Walker's (1991) $400 \mathrm{~km} \mathrm{~s}^{-1}$ (from highdispersion spectra) than to the often quoted $230 \mathrm{~km} \mathrm{~s}^{-1}$. For $i=44^{\circ}$, this leads to $v_{\text {rot }}=575 \mathrm{~km} \mathrm{~s}^{-1}$. The upper limit to the rotational period is then $0.55-$ $0 \mathrm{~d} 71\left(0.79-1 . \mathrm{d} 02\right.$ for $\left.i=90^{\circ}\right)$.

$\varphi \operatorname{Per}(H R$ 496, HD 10516) is a well-known binary with $P=126.6731$ (Božić et al. 1995). The secondary is a hot O subdwarf - see Gies et al. (1998). Quirrenbach et al. (1997) give $i>63^{\circ}$. According to Gies et al. (1998) $M_{1} \sin ^{3} i$ 
Table 3. Radii of emission-line stars which are members of open clusters or associations with good mean Hipparcos parallaxes and radii of some field emission-line stars, based on their individually measured parallaxes: $R_{1}$ denotes the radius (in $R_{\odot}$ ) derived using $\log T_{\text {eff }}, V_{0}$, and mean (cluster) or individual $\pi$. The ranges of possible radii, estimated from the errors of the parallaxes, are given in brackets

\begin{tabular}{rrrrrc}
\hline Star & HD & $\log T_{\text {eff }}$ & $V_{0}$ & $M_{\text {bol }}$ & $R_{1}$ \\
\hline HR 1051 & 21551 & 4.097 & 5.560 & -1.632 & $4.04(3.90-4.19)$ \\
$\psi$ 37 Per & 22192 & 4.217 & 3.910 & -4.081 & $7.18(6.93-7.45)$ \\
17 Tau & 23302 & 4.114 & 3.580 & -2.683 & $6.06(5.91-6.22)$ \\
23 Tau & 23480 & 4.132 & 4.060 & -2.341 & $4.77(4.63-4.91)$ \\
$\eta$ 25 Tau & 23630 & 4.097 & 2.750 & -3.409 & $9.16(8.89-9.44)$ \\
BU 28 Tau & 23862 & 4.063 & 4.880 & -1.082 & $3.67(3.56-3.78)$ \\
MX 48 Per & 25940 & 4.235 & 3.480 & -4.609 & $8.43(8.14-8.75)$ \\
HR 1761 & 34959 & 4.190 & 6.338 & -2.726 & $4.36(4.16-4.57)$ \\
V1086 25 Ori & 35439 & 4.418 & 4.798 & -5.427 & $5.29(5.05-5.55)$ \\
V1372 Ori & 36012 & 4.190 & 7.168 & -1.896 & $2.97(2.84-3.12)$ \\
HD 37330 & 37330 & 4.149 & 7.331 & -1.459 & $2.94(2.80-3.08)$ \\
HR 2968 & 61925 & 4.140 & 5.717 & -3.150 & $6.67(6.29-7.10)$ \\
PP Car & 91465 & 4.300 & 3.150 & -4.774 & $6.74(6.58-6.91)$ \\
$\mu$ Cen & 120324 & 4.301 & 3.500 & -4.252 & $5.28(5.20-5.35)$ \\
$\eta$ Cen & 127972 & 4.350 & 2.229 & -4.919 & $5.72(5.31-6.21)$ \\
\hline o 22 Cas & 4180 & 4.190 & 4.290 & -4.363 & $9.26(7.54-11.99)$ \\
$\gamma 27$ Cas & 5394 & 4.519 & 2.700 & -6.811 & $6.28(5.69-7.02)$ \\
$\varphi 42$ And & 6811 & 4.097 & 4.032 & -3.529 & $9.68(8.20-11.81)$ \\
$\varphi$ Per & 10516 & 4.430 & 3.820 & -5.550 & $5.30(4.55-6.34)$ \\
13 Tau & 23016 & 4.090 & 5.440 & -.802 & $2.85(2.52-3.26)$ \\
$\xi 46$ Per & 24912 & 4.468 & 3.190 & -8.348 & $16.13(11.68-26.03)$ \\
$\lambda 47$ Per & 25642 & 3.985 & 4.226 & -1.118 & $5.34(4.90-5.86)$ \\
$\lambda$ 69 Eri & 33328 & 4.373 & 4.130 & -6.899 & $12.82(8.70-24.33)$ \\
$\zeta 123$ Tau & 37202 & 4.418 & 2.670 & -5.460 & $5.37(4.75-6.18)$ \\
$\omega 28$ CMa & 56139 & 4.233 & 4.000 & -4.940 & $9.91(8.51-11.86)$ \\
V744 88 Her & 162732 & 4.149 & 6.660 & -2.328 & $4.38(3.66-5.47)$ \\
V2048 66 Oph & 164284 & 4.364 & 4.370 & -4.549 & $4.53(3.90-5.40)$ \\
V1661 55 Cyg & 198478 & 4.420 & 2.870 & -8.930 & $26.30(19.07-42.37)$ \\
V1931 60 Cyg & 200310 & 4.431 & 5.270 & -5.503 & $5.16(4.15-6.81)$ \\
$o 1$ And & 217675 & 4.190 & 3.890 & -4.180 & $8.51(7.45-9.92)$ \\
\hline & & & & & \\
\hline
\end{tabular}


$=8.90 \mathrm{M}_{\odot}$. If the equatorial plane of the primary is coplanar with the orbital plane, one obtains: $8.9 \leq M_{1} \leq 12.6 \mathrm{M}_{\odot}$. My best estimate gives a position in the $\log R$ vs. $\log T_{\text {eff }}$ diagram which corresponds to a normal star of about $12 \mathrm{M}_{\odot}$. This is a very satisfactory agreement.

$\mu$ Cen (HR 5193, HD 120324) is a very intensively studied B2e star (cf., e.g., Rivinius et al. 1998). My values of $M=7.5 \mathrm{M}_{\odot}$ and $R=5.2-5.4 \mathrm{R}_{\odot}$, in combination with $v$ sin $i=130 \mathrm{~km} \mathrm{~s}^{-1}$ (Brown and Verschueren 1997) give an upper limit to the rotational period of 2.06 .

$\eta$ Cen (HR 5193, HD 120324) is another intensively studied B2e star, known for rapid changes with a period of 0.64. de Zeeuw et al. (1999) admit that its peculiar space motion may be due to unrecognized duplicity and that it may be a member of the Upper Centaurus Lupus. If it is a member (which is improbable), then $R=8.36-8.59 \mathrm{R}_{\odot}$. If it is a non-member, I obtain $R=5.31-6.21 \mathrm{R}_{\odot}$. Brown and Verschueren (1997) give $v \sin i=260 \mathrm{~km} \mathrm{~s}^{-1}$ while Štefl et al. (1995) obtained $v \sin i=335 \mathrm{~km} \mathrm{~s}^{-1}$ from high-dispersion spectra. For the latter value, an upper limit to the rotational period is $0 \mathbf{d} 80-0 \mathbf{d} 93$.

V1661 Cyg (55 Cyg, HD 198478) is a reddened star, usually classified B3Iae. Comerón et al. (1998) conclude that a nearby B1III star HD $198479=$ HIP 102722 belongs to the same association. I therefore used its $E(B-V)=0.66$ to deredden V1661 Cyg. One obtains a large radius $\left(20-70 R_{\odot}\right)$ but the result is uncertain because of a very uncertain parallax of $\mathrm{HD} 198479$.

$o$ And (HR 8762, HD 217675) is a multiple system of stars (Hill et al. 1989). The primary is a B5IIle star with well-known rapid light variations with a period of 1.57. I obtained $R=8.51(7.45-9.92) R_{\odot}$ and an evolutionary mass of $6.5 \mathrm{M}_{\odot}$, in a good agreement with an independent estimate by Hill et al. (1989). This implies a star evolved away from the main sequence. For $v \sin i=260 \mathrm{~km} \mathrm{~s}^{-1}$, an upper limit to the rotational period is $1.66\left(1.45-1{ }^{\mathrm{d}} 93\right)$. The observed period is very close to this limit.

\section{What is the evolutionary stage of Be stars?}

It is important to realize that - depending on the true origin of Be envelopes - this may or may not be a legitimate question. Let us consider two of the suggested scenarios for the formation of the envelopes: In the compressed wind model (Bjorkman \& Cassinelli 1993) the envelope originates via a physical process from the material of the dynamical stellar atmosphere of the underlying star. If so, then the formation and properties of the envelopes should be somehow related to the evolutionary stage of the stars in question. This is not true if the envelope is formed by a gas inflow from the companion of the Be star in a binary system as assumed in the binary model (Kr̃́ž and Harmanec 1975). In such a case, the properties of the envelope are given basically by the evolutionary stage of the mass losing star and by geometry of the binary orbit, not by the evolutionary stage of the Be star itself.

\subsection{Comparison with evolutionary models}

Slettebak (1985) made the most systematic study of Be stars in open clusters and concluded that $\mathrm{Be}$ stars may exist in various evolutionary stages, including the unevolved stage near the zero-age main sequence. To see what the new data 

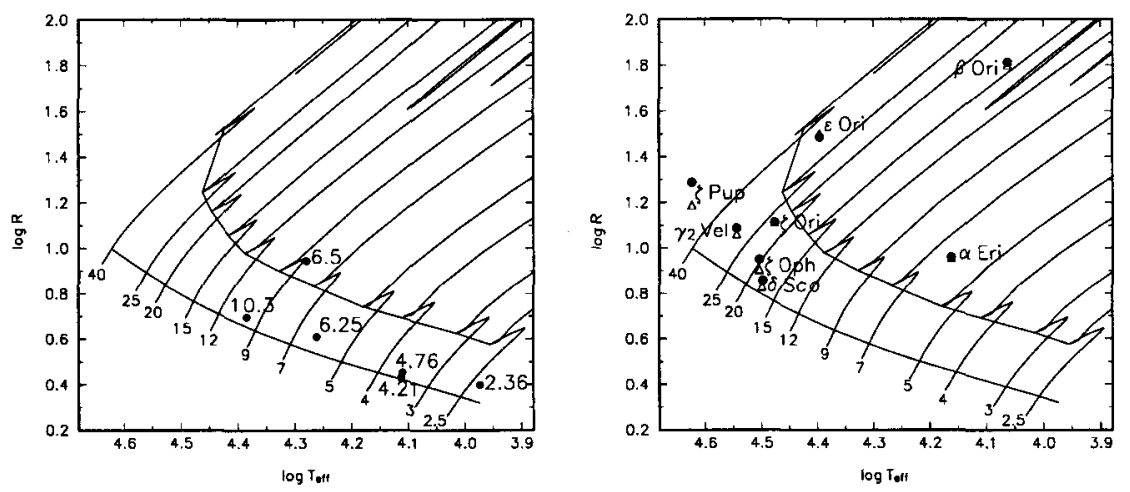

Figure 2. Left: Radii of Be stars which are members of eclipsing binaries are plotted vs. $T_{\text {eff. }}$ For comparison, the evolutionary tracks of Schaller et al. (1992) for stars of 2.5 to $40 \mathrm{M}_{\odot}$ are also shown. Normal values of magnitudes and radii for detached binaries, derived by Harmanec (1988), are shown by a line. Another line denotes the terminal main sequence. Mass of each star is also given. Right: Comparison of radii of Be stars derived from the Hipparcos parallaxes and their measured angular radii (black circles) with the radii based on the observed $V_{0}, T_{\text {eff }}$ and the Hipparcos parallaxes (triangles). Individual emission-line stars are identified. Other notation is the same

show, I compared the observed properties of some bright Oe and Be stars with those derived from well observed detached binaries (as compiled by Harmanec 1988 ) and also with the evolutionary tracks for new opacities and $Z=0.02$ published by the Geneva group (Schaller et al. 1992) which include mass loss for more massive models. Fig. 2 (left) shows the $\log R$ vs. $T_{\text {eff }}$ diagram for Be stars in eclipsing binaries with reliably derived physical properties. In Fig. 2 (right), the radii of those emission-line stars for which angular-diameter measurements were given by Code et al. (1976) are compared with radii based on the observed radiative properties of respective stars. Figure 3 compares the radii of Be stars which are members of open clusters and associations, and for which accurate mean Hipparcos cluster parallaxes could be used, with the radii derived for the field emission-line stars.

One can make the following tentative conclusions from these diagrams:

1. There are some Be stars having masses and radii similar to normal B stars but no star close to ZAMS was found in the present limited sample of bright stars. If confirmed from a much larger sample, this could support the recent conclusions by Fabregat \& Torrejón (2000) who concluded that the Be phenomenon can only occur during the second half of the main-sequence lifetime of $B$ stars.

2. For three of the six eclipsing binaries there is a significant discrepancy between the directly derived mass and the evolutionary mass. This may be a consequence of the large-scale mass exchange in these particular systems. Such cases may also be present among other Be stars.

3. There is no clear distinction between luminosity V-III and II-Ia Be stars: the emission-line objects exist both at MS phases of the core hydrogen burning 

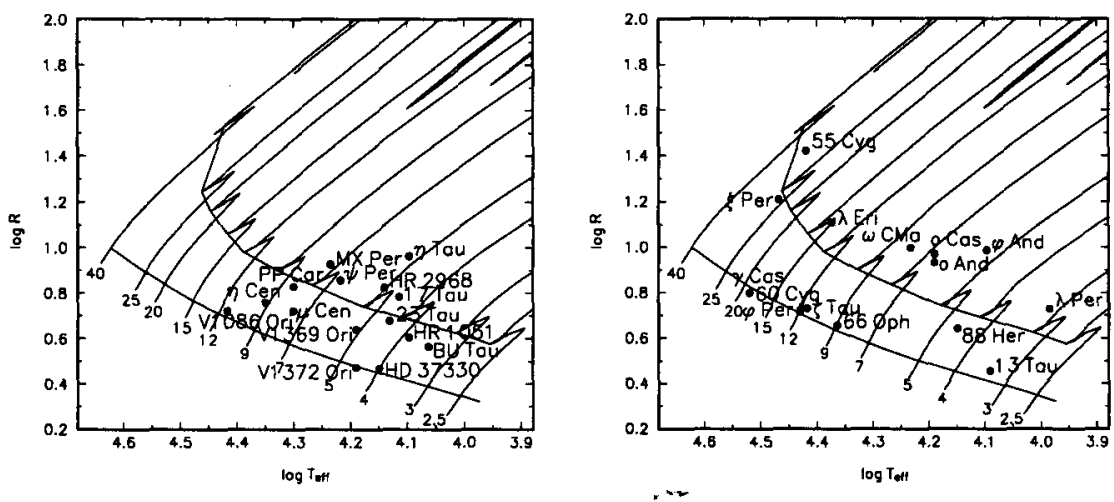

Figure 3. Left: Radii of Be stars which are members of open clusters are plotted vs. $T_{\text {eff }}$. For comparison, the evolutionary tracks of Schaller et al. (1992) for stars of 2.5 to $40 \mathrm{M}_{\odot}$ are also shown. Normal values of magnitudes and radii for detached binaries, derived by Harmanec (1988), are shown by a line. Another line denotes the terminal main sequence. Right: Radii of field emission-line stars derived from their individually measured Hipparcos parallaxes are shown

and in later evolutionary stages. The transition is quite smooth and the term "classical Be stars" should perhaps be abandoned. Note that even some stars spectrally classified V-III seem to have radii larger than what would correspond to main-sequence objects. This is, for instance, the case of $\eta$ Tau, HR 2968 or $\omega \mathrm{CMa}$. One possible explanation is that such stars are in fact unrecognized binaries or even multiple systems. Note, however, that the assumption of two equally bright stars instead of one would decrease their $\log R$ by only 0.15 dex.

\subsection{Metallicity effects}

Maeder et al. (1999) investigated early- $B$ emission-line stars in well studied galactic clusters and in LMC and SMC. The fraction of Be stars among B stars was found to increase with decreasing $Z$. They conclude that the higher the average rotation speed may be the lower the metallicity.

While the effect reported is undoubtedly real I feel that further studies should also investigate the relative percentage of Be stars among $B$ stars as a function of the spectral subclass. There are indications that the fraction of $\mathrm{Be}$ stars along the B sequence has different maxima in different clusters. There is usually also another maximum near B8. The long-term variability of Be stars discussed above is also an important factor: note that the method of selection used by Maeder et al. prefers Be stars which are at phases of strong emission, i.e. those which have their observed $V$ magnitudes brighter in most cases than what would correspond to the underlying star without an envelope.

Acknowledgments. Dr. J.R. Percy kindly put his individual $U B V$ observations of Be stars at my disposal. Useful comments on the matter were kindly communicated to me by Dr. J. Dachs. I am also grateful to Drs. D. Holmgren and M.A. Smith for their comments and a careful proofreading of the draft ver- 
sion of this text. Drs. J. Fabregat, E. Grebel and A. Maeder kindly informed me about their recent results prior to publication. I apologize to all colleagues whose work could not be mentioned explicitly because of a limited space. The support from the grant A3003805 of the GA AV ČR and from the organizers of the Alicante conference is gratefully acknowledged.

\section{References}

Batten, A.H. 1974, Publ. Dom. Astrophys. Obs., 14, 191

Bedding, T.R. 1993, AJ, 106, 768

Beeckmans, F., \& Hubert-Delplace, A.M. 1980, A\&A, 86, 72

Bell, S.A., Kilkenny, D., \& Malcolm, G.J. 1987, MNRAS, 226, 879

Bjorkman, J.E., Cassinelli, J.P. 1993, ApJ, 409, 429

Božić, H., Harmanec, P., Horn, J., Koubský, P., Scholz, G., McDavid, D., Hubert, A.-M., \& Hubert, H. 1995, A\&A, 304, 235

Brown, A.G.A., \& Verschueren, W. 1997, A\&A, 319, 811

Code, A.D., Davis, J., Bless, R.C., \& Hanbury Brown, R. 1976, ApJ, 203, 417

Collins II, G.W, Truaux, R.J., \& Cranmer, S.R. 1991, ApJS, 77, 541

Comerón, F., Torra, J., \& Gómez, A.E. 1998, A\&A, 330, 975

Dachs, J. 1982, in IAU Symp. 98: Be Stars, ed. by Jaschek, M., \& Groth, H.-G.,

D. Reidel, Dordrecht, 19

de Zeeuw, P.T., Hoogerwerf, R., de Bruijne, J.H.J., Brown, A.G.A., \& Blaauw A. $1999, \mathrm{AJ}, 117,354$

Fabregat, J., \& Torrejón, J.M. 2000, A\&A, 357, 451

Gies, D.R., Bagnuolo Jr., W.G., Ferrara, E.C., Kaye, A.B., Thaller, M.L., Penny,

L.R., \& Peters, G.J. 1998, ApJ, 493, 440

Hadrava, P. 1992, A\&A, 256, 519

Hadrava, P. 1995, A\&AS, 114, 393

Harmanec, P. 1983, Hvar Obs. Bull., 7, 55

Harmanec, P. 1988, Bull. Astron. Inst. Czechosl. 39, 329

Harmanec, P. 1989, Bull. Astron. Inst. Czechosl. 40, 201

Harmanec, P. 1994, in NATO ARW: The Impact of Long-term Monitoring on

Variable Star Research, Ed. by C. Sterken and M. de Groot, Kluwer,

Dordrecht, 55

Harmanec, P. 1998a, A\&A, 334, 558

Harmanec, P. 1998b, A\&A, 335, 173

Hill, G.M., Walker, G.A.H., Yang, S., \& Harmanec, P. 1989, PASP, 101, 258

Hill, G., Harmanec, P., Pavlovski, K., Božić, H., Hadrava, P., Koubský, P., \& Żižñovský, J. 1997, A\&A, 324, 965

Hirata, R. 1995, PASJ, 47, 195

Howarth, I.D. 1979, J. Brit. Astron. Assoc., 89, 378

Hubert, A.-M., Floquet, M. 1998, A\&A, 335, 567

Hutchings, J.B. 1970, MNRAS, 150, 55 
Koubský, P., Harmanec, P., Kubát, J., Hubert, A.-M., Božić, H., Floquet, M., Hadrava, P., Hill, G., Percy, J.R. 1997, A\&A, 328, 551

Koubský, P., Harmanec, P., Hubert, A.-M., Floquet, M., Kubát, J., Yang, S., Holmgren, D., Ballereau, D., Božić, H., Cao, H., Chauville, J., Eenens, P., Huang, L., \& Percy, J.R. 2000, A\&A, 356, 913

Kř́iž, S., \& Harmanec, P. 1975, Bull. Astron. Inst. Czechosl., 26, 65

Maeder, A., Grebel, E.K., \& Mermilliod, J.-C. 1999, A\&A, 346, 459

Maxted, P.F.L., Hill, G., \& Hilditch, R.W. 1995, A\&A, 301, 135

Moon, T.T., \& Dworetsky, M.M. 1985, MNRAS, 217, 305

Olson, E.C. 1984, PASP, 96, 162

Olson, E.C., \& Plavec, M.J. 1997, AJ, 113, 425, ..

Pavlovski, K., Harmanec, P., Božić, H., Koubský, P., Hadrava, P., Kříž, S., Ružić, Ž., \& Štefl, S. 1997, A\&AS, 125, 75

Perryman, M.A.C., Høg, E., Kovalevsky, J., Lindegren, L., \& Turon, C. 1997, ESA SP-1200: The Hipparcos and Tycho Catalogues

Piccioni, A., Bartolini, C., Bernabei, S., Galleti, S., Guarnieri, A., \& Valentini, G. 2000, This Volume, p.585

Pinsonneault, M.H., Stauffer, J., Soderblom, D.R., King, J.R., \& Hanson, R.B. 1998, ApJ, 504, 170

Plavec, M.J., \& Dobias, J.J. 1987, AJ, 93, 440

Poeckert, \& Marlborough, M. 1978, ApJ, 220, 940

Rivinius, Th., Baade, D., Štefl, S., Stahl, O., Wolf, B., \& Kaufer, A. 1998, A\&A, 336,177

Quirrenbach, A., Bjorkman, J.E., Hummel, C.A., Buscher, D.F., Armstrong, J.T., Mozurkewich, D., Elias, N.M., II., \& Babler, B.L. 1997, ApJ, 479, 477

Robichon, N., Arenou, F., Mermilliod, J.-C., \& Turon, C. 1999, A\&A, 345, 471 Roche, P., Tarasov, A.E., Clark, S., Lyuty, V., \& Larionov, V. 2000, This Volume, p.589

Schaller, G., Schaerer, D., Meynet, G., \& Maeder, A. 1992 A\&AS, 96, 269

Slettebak, A. 1982, ApJS, 50, 55

Slettebak, A. 1985, ApJS, 59, 769

Stettebak, A. 1987, in IAU Col. 92: Physics of Be stars, Ed. by A. Slettebak and T.P. Snow, 24

Štefl, S., Baade, D., Harmanec, P., \& Balona, L.A. 1995, A\&A, 294, 135

Tomkin, J. 1981, ApJ, 244, 546

van Leeuwen F. 1999, A\&A, 341, L71

Walker, G.A.H. 1991, in Rapid Variability of OB Stars: Nature and Diagnostic Value, ed. by D. Baade, ESO Conf. Proc. 136, 27 


\section{Discussion}

H. Henrichs: I would not recommend to abandon the term classical Be stars as you suggest, because the B supergiants always show Balmer emission (admittedly variable) in contrast to $\mathrm{Be}$ stars. In supergiants the presence of emission is understood because of their extended atmosphere, but in low-luminosity B stars the intermittent presence of an extended atmosphere remains a surprise.

P. Harmanec: I am not implying that there are no genuine supergiants produced by normal stellar evolution away from the main sequence. What I am trying to remind repeatedly is that one should be aware of the phenomenological definition of Be stars and investigate both, the variability patterns and basic physical properties of individual objects very carefully. There may be, and I am tempted to say there surely are cases when very large and extended $\mathrm{Be}$ envelopes simulate objects quite reminiscent of genuine supergiants. As I have demonstrated in my talk, there are classical Be stars which have radii placing them away from the main sequence, and probably also Be supergiants with radii of main-sequence stars. Possibly, the transition between low-luminosity and high-luminosity Be stars is quite smooth.

M. Smith: I now believe that the $v \sin i$ of $\gamma$ Cas is close to $230 \mathrm{~km} \mathrm{~s}^{-1}$, as given by Slettebak (1982). Based on additional work on $\gamma$ Cas I now believe that my own previous work on $v \sin i$ on this star and Walker's (1991) is erroneous because we were measuring the acceleration on line profiles of migrating subfeatures. These features are probably due to passage in front of the star of circumstellar structures corotating at the star's angular rotation rate. Shortly before his death, I spoke with Arne Slettebak about his quoted value $v \sin i$ $=230 \mathrm{~km} \mathrm{~s}^{-1}$. He "stuck to his guns" in favour of his published value. In addition, David Gray has carefully observed the He II 4686 line with his high $\mathrm{S} / \mathrm{N}$ ratio-capable Reticon system. (This is a "hot ion line" not easily influenced by disk/shell contamination.) He tells me from his data that he also derives a value of $v \sin i$ of about $200 \mathrm{~km} \mathrm{~s}^{-1}$.

P. Harmanec: This problem has several aspects. First, if I am right with the concept of varying pseudophotosphere, then it is necessary to realize that such a pseudophotosphere has been present in $\gamma$ Cas all the time since the forties. It means that the width and depth of supposedly photospheric lines may vary secularly with time. Another aspect worth mentioning is the following: For another hot line-profile variable, $\zeta .0 \mathrm{ph}$, I measured accelerations of sub-features of different lines, including lines of high ionization like He II 4686 in Walker's et al. high-S/N Reticon spectra (Harmanec 1989). I found an amplitude corresponding to $v \sin i$ of $420 \mathrm{~km} \mathrm{~s}^{-1}$ for the hydrogen and other lower-ionization lines but only something like $360 \mathrm{~km} \mathrm{~s}^{-1}$ for the He II 4686 line. This was then fully confirmed by another measuring technique by Walker (1991). One possible interpretation (which also Walker adopted) is that cooler lines measure the rotation rate at the equator while the lines of higher ionization show the linear rotation rate of regions at higher latitudes. All this shows how complicated problem we are facing here. I am sure that a useful exercise would be to compare the observed high-dispersion profiles with a homogeneous set of theoretical profiles, to estimate corresponding $v \sin i, T_{\text {eff }}$ and $\log g$ and to see possible time and line-to-line evolution. The profiles which I have seen are certainly much broader than what would correspond to $230 \mathrm{~km} \mathrm{~s}^{-1}$. 